\title{
Oxidized cellulose: an unusual cause of post hysterectomy hemorrhage
}

\author{
Amrita*, Sandhya Jain, Swati Sharma, Shalini Rajaram, Bindiya Gupta
}

\begin{abstract}
Department of Obstetrics and Gynecology, University College of Medical Sciences and Guru Teg Bahadur Hospital, Delhi-110095, India
\end{abstract}

Received: 14 June 2016

Accepted: 02 July 2016

\section{*Correspondence:}

Dr. Amrita,

E-mail: amrita4dec@gmail.com

Copyright: (c) the author(s), publisher and licensee Medip Academy. This is an open-access article distributed under the terms of the Creative Commons Attribution Non-Commercial License, which permits unrestricted non-commercial use, distribution, and reproduction in any medium, provided the original work is properly cited.

\begin{abstract}
Haemostatic material made of oxidized cellulose is a bio-absorbable fabric used widely for intraoperative haemostasis and adhesion prevention in surgery. The knit mesh facilitates platelet adhesion and aggregation during surgery. However, rarely it may cause a foreign-body reaction, which is indistinguishable from abscess or granuloma formation. This report describes a case of a local tissue reaction due to oxidized regenerated cellulose followed by vault erosion and vaginal bleeding, mimicking pelvic sepsis post hysterectomy. The signs and symptoms resolved completely following removal of the oxidized cellulose fabric.
\end{abstract}

Keywords: Oxidized cellulose, Haemostatic material, Hysterectomy, Hemostasis

\section{INTRODUCTION}

Haemostatic material made of oxidized cellulose is a bioabsorbable fabric used widely for intraoperative haemostasis and adhesion prevention in surgery. The knit mesh facilitates platelet adhesion and aggregation during surgery. ${ }^{2}$ However, rarely it may cause a foreignbody reaction, which is indistinguishable from abscess or granuloma formation. This report describes a case of a local tissue reaction due to oxidized regenerated cellulose followed by vault erosion and vaginal bleeding, mimicking pelvic sepsis post hysterectomy. The signs and symptoms resolved completely following removal of the oxidized cellulose fabric.

\section{CASE REPORT}

A 46-year-old $\mathrm{P}_{2} \mathrm{~L}_{2}$ with a diagnosis of abnormal uterine bleeding underwent total abdominal hysterectomy with bilateral salpingo-oophorectomy in our hospital. She had extensive pelvic and abdominal adhesions and chocolate cyst in left ovary. Haemostatic fabric made of oxidized cellulose was placed at the vaginal cuff at completion of the surgery to achieve haemostasis.
Patient's immediate postoperative course was uneventful and she was discharged on fourth day. She presented to the outpatient department after 3 months with complaints of blood mixed discharge per vaginum and pelvic pain since the time of discharge. She was afebrile and her vitals were stable. Abdominal examination showed no abnormality. On per speculum examination, there was foul smelling blood mixed discharge and mesh like material at the vault. On per vaginal examination, there was gritty foreign body sensation at vault along with mild tenderness. A complete blood count, basic metabolic profile and urinalysis were performed, and all the findings were within normal limits. Chest and pelvic radiographs were unremarkable. Urine and high vaginal swab cultures yielded no growth. Ultrasound of the pelvis showed expected postsurgical findings along with an echogenic focus at the vaginal cuff, however there was no fluid collection, abscess or hematoma. The patient was admitted and treated with intravenous antibiotics. She underwent examination under anaesthesia. There was oxidized cellulose fabric eroding through the vault and impacted in the adjacent tissue (Figure 1). It was removed in piecemeal and haemostatis secured by tight packing. Her symptoms resolved subsequently over the next 2-3 days. She was discharged without need of any further 
treatment and scheduled for follow-up in outpatient department. On follow up, the patient was relieved of her symptoms and healthy granulation tissue was seen at the vault (Figure 2).

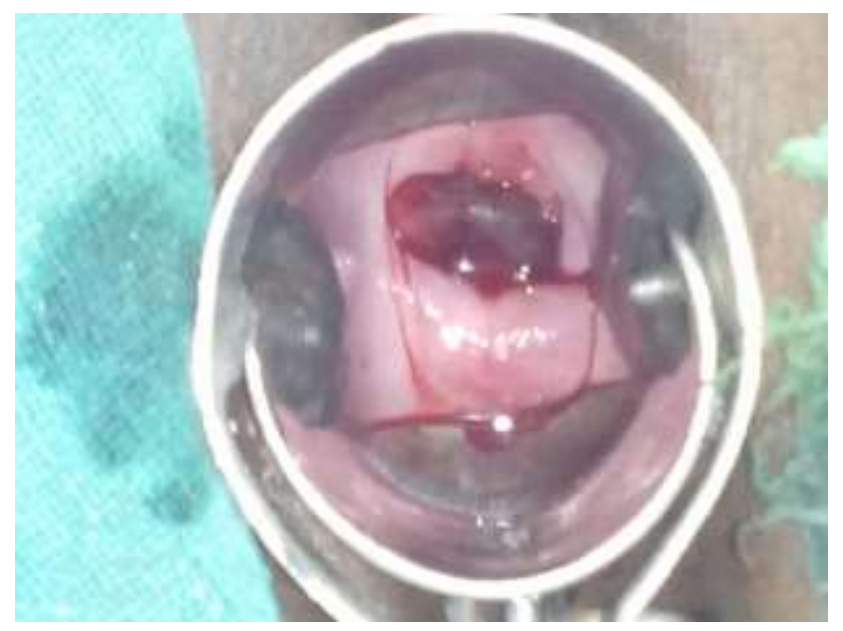

Figure 1: Clinical photograph showing oxidized cellulose fabric eroding through the vault.

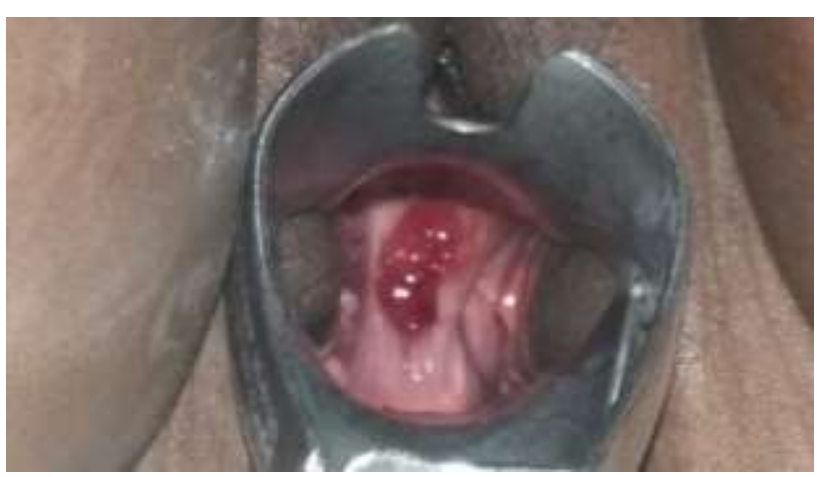

Figure 2: Clinical photograph showing healthy granulation tissue on follow-up after 2 weeks.

\section{DISCUSSION}

Topical haemostatic agents are being commonly used in gynaecologic surgery, especially in the setting of laparoscopic procedures. Haemostatic material made of oxidized cellulose facilitates the production of an artificial clot. ${ }^{3,4}$ Although the mechanism of action is not fully understood, oxidized cellulose forms a gelatinous mass when saturated with blood. It acts as a matrix for fibrin deposition, promoting platelet plug formation and aiding surgical haemostasis. It also causes local vasoconstriction; further controlling intraoperative bleeding. ${ }^{5}$ Haemostatic material made of oxidized cellulose may be removed or left in situ depending on the clinical need. If left in situ degradation is expected to commence within $24-48 \mathrm{~h}$ and will be surrounded by inflammatory granulation tissue at day 7; complete degradation usually occur between 4 and 8 weeks. ${ }^{6,7}$ Therefore patients with oxidized cellulose placed during surgery may present postoperatively with the haemostatic material still present.

This is a rare case of a local tissue reaction to oxidized cellulose mimicking pelvic sepsis. Oxidized cellulose has been reported to present as pseudo cancer; abscess and granuloma formation. ${ }^{2-4}$ The bulk of these cases presented with pain or on routine follow up imaging between 6 and 52 weeks. ${ }^{1-4,6-9}$ Previously a study reported the cases of 3 patients who underwent oxidized regenerated cellulose placement during laparoscopic gynaecologic surgery. ${ }^{2}$ They subsequently presented with signs and symptoms resembling an abscess. CT scan was used to differentiate between the characteristics of oxidized regenerated cellulose and those of abscess formation on the vaginal cuff and the cases were managed accordingly. Ibrahim et al reported a whitish thick cheesy material with the appearance of pus with pieces of non-absorbed oxidized cellulose one year following surgery. ${ }^{8}$ The cultures on this material turned out to be sterile with no organisms seen or grown.

No mortalities have been reported so far and conservative management has been sufficient even in cases with worrying symptoms. ${ }^{9}$ It is important to document in notes when oxidized cellulose is used despite typical radiological appearances being reported. ${ }^{7}$ Some guidelines recommend using it sparingly where its use is indispensible, as many such cases have been attributed to use in excess. ${ }^{8}$ The Society of Obstetricians and Gynaecologists of Canada recommends using oxidized cellulose for patients who are at high risk of forming clinically significant adhesions. However, ironically the risk of adhesions may increase if optimal hemostasis is not achieved.

The removal of oxidized cellulose leads to complete resolution of symptoms confirming it to be the cause of secondary hemorrhage in our case. We continue to use oxidized cellulose in small quantities despite possibility of such reaction, as its benefits far outweigh the risks. It remains important for surgeons and radiologists to be aware of its possible complications despite rare incidence.

\section{Funding: No funding sources Conflict of interest: None declared Ethical approval: Not required}

\section{REFERENCES}

1. Royds J, Kieran S, Timon C. Oxidized cellulose (Surgicel) based reaction post thyroidectomy mimicking an abscess: A case report. Int J Surg Case Rep. 2012;3(7):338-9.

2. Tam T, Harkins G, Dykes T, Gockley A, Davies M. Oxidized Regenerated Cellulose Resembling Vaginal Cuff Abscess. J Soc Laparoendosc Surg. 2014;18(2):353-6. 
3. Somani BK, Kasthuri RS, Shave RM, Emtage LA. Surgicel granuloma mimicking a renal tumour. Surgery. 2006;139(3):451.

4. Salmo E, Bisset R, Aldean I. Oxidized cellulose mimicking a recurrent colorectal cancer: report of a case and review of the literature. Colorectal Dis. 2010;12(4):381-2.

5. Hazarika EZ. Oxidized regenerated cellulose: an effective emergency hemostatic in burns surgery. $\mathrm{Br}$ J Plast Surg. 1985;38:419-21.

6. Agarwal MM, Mandal AK, Agarwal S, Lal A, Prakash M, Mavuduru R. Surgicel granuloma: unusual cause of "recurrent" mass lesion after laparoscopic nephron-sparing surgery for renal cell carcinoma. Urology. 2010;76(2):334-5.

7. Alpaslan C, Alpaslan GH, Oygur T. Tissue reaction to three subcutaneously implanted local hemostatic agents. Br J Oral Maxillofac Surg. 1997;35(2):12932.

8. Ibrahim MF, Aps C, Young CP. A foreign body reaction to Surgicel mimicking an abscess following cardiac surgery. Eur J Cardiothorac Surg. 2002;22(3):489-90.

9. Arnold A, Sodickson A. Postoperative Surgicel mimicking abscesses following cholecystectomy and liver biopsy. Emerg Radiol. 2008;15:183-5.

Cite this article as: Amrita, Jain S, Sharma S, Rajaram S, Gupta B. Oxidized cellulose: an unusual cause of post hysterectomy hemorrhage. Int J Reprod Contracept Obstet Gynecol 2016;5:2866-8. 